\title{
26
}

\section{The spatial GOLF project}

\author{
S. TURCK-CHIEZE \\ DAPNIA/Service d'Astrophysique, CE Saclay, 91190 Gif sur Yvette, FRANCE
}

and the GOLF scientific and technical team

R. BOCCHIA ${ }^{5}$, P. BOUMIER ${ }^{1}$, M. CANTIN ${ }^{2}$, J. CHARRA $^{1}$, B. COUGRAND $^{1}$, J. CRETOLLE ${ }^{2}$, N. DENIS ${ }^{2}$, R. DUC ${ }^{2}$, H.

DZITKO $^{2}$, M. DECAUDIN ${ }^{1}$, A. GABRIEL ${ }^{1}$, J. HERREROS $^{3}$, G.

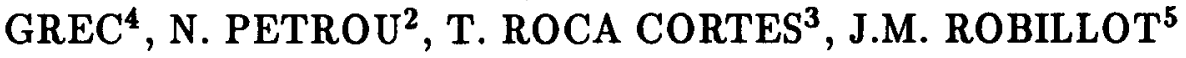

${ }^{1}$ IAS, Université Paris XI, Bat 121, 91405 ORSAY Cedex, FRANCE

2 DAPNIA/Service d'Astrophysique, CE Saclay, 91190 Gif sur Yvette, FRANCE

3 Instituto Astrofisica de Canarias, Tenerife, SPAIN

4 Dép. d'Astrophysique, U'niversité de Nice, 06108 Nice Cedex 02, FRANCE

- Observatoire de Bordeaux, BP 89, 99270 FLOIRAC, FRANCE

\section{Abstract}

This spatial experiment is under construction and has been defined as a 2 years mission on board SOHO, a satellite dedicated to the Sun which will be launched in mid 95. The main objectives are the detection of solar low degree acoustic modes and solar gravity modes for improving our knowledge of the solar nuclear region.

\subsection{Introduction}

The spatial experiment, GOLF (Global oscillations at Low Frequencies), has been accepted by ESA in March 1988 and should be boarded on the SOHO (SOlar and Heliospheric Observatory) satellite (Damé et al 1988, Gabriel et al 1989). This satellite will be launched by NASA in mid 1995 . The objectives of this experiment is to enhance our knowledge of the solar interior by the measurement of the low degree acoustic modes: $1=0,1,2$, 3 , i.e the most penetrating ones, and by the possible measurement of the gravity modes. These different types of modes correspond to frequencies between some $10^{-6}$ and $810^{-3} \mathrm{~Hz}$. On the same satellite there will be two other helioseismic experiments: VIRGO and MDI, the first one is a variability solar irradiance measurement in different wavelengths which allows to reach acoustic modes of degree $l=0,7$. The second one, using a Michel- 
son Doppler imager, is a complementary experiment which must be able to detect degree acoustic modes up to $1=4500$.

The success of such mission is largely dependent on the stability of the measurements, which requires a pointing stability of the satellite better than 1 arc sec per 15 minutes.

\subsection{The low degree acoustic modes}

With GOLF instrument, we hope to improve our knowledge on the absolute values of the low degree acoustic mode frequencies and on the variability and the width of these modes which will be followed with a duty cycle of nearly $100 \%$ during the total mission. If it is the case, one will better determine the solar sound speed, density, composition and rotation in the nuclear region and one will progress on the excitation of these modes. Presently, the sound speed extracted from the acoustic mode frequencies is well defined down to $0.3 R \odot$, the 2700 detected acoustic modes have already allowed to reject some physical idea as large mixing or hypothetical particles as WIMPs (Turck-Chièze et al 1993), moreover, they have pushed us to improve the microscopic physics used in standard model, particularly the solar equation of state and the opacity coefficients. Doing so, one has significantly improved the intermediate region between the nuclear region and the convective outerzone (Turck-Chièze and Lopes 1993). Concerning the central region, we have shown the low sensitivity to such ingredients due to the luminosity constraint, but the important role of some specific reaction rates and of the screening effect in different parts of the calculation (see Dzitko et al these proceedings for the screening effect on the reaction rates). The central solar region is not presently well understood due to the unsufficient number of detected modes. One aim of the GOLF experiment is to better determine tha acoustic modes corresponding to frequencies between $10^{-3}$ to $210^{-3} \mathrm{~Hz}$, in order to put some constraints on the neutrino fluxes emitted by this solar region and to confirm or infirm the possible mixing and central convection suggested by the present analysis (Gough and Kosovishev 1993, Turck-Chièze, Dzitko and Lopes 1993).

To progress in the knowledge of this solar region, the spatial GOLF experiment presents several advantages: the satellite SOHO will be placed at the L1 Lagrange point, located at 1 million $\mathrm{km}$ from the earth. This situation suppresses effects of variability of the atmosphere due to large clouds and due to the connection between different sites of a ground network. This situation allows also a good continuity of the data, without day night or season variations, moreover, the duty cycle will be improved by the adjonction 
of an on board memory to avoid discontinuities due to bad communication with antenna. One hopes to reach a great stability of the instrument (at a level of $10^{-6}$ ), first by a uniform temperature inside the instrument always precisely pointed towards the Sun, secondly, by high counting rate photomultipliers which must garantee a white noise of very low level in order to extract a velocity of about $1 \mathrm{~mm} / \mathrm{s}$ after three weeks of continuous experiment and finally because the experiment is mainly sensitive to unstability inside the cycle of the experiment which is lower than $40 \mathrm{~s}$.

\subsection{The research for gravity modes}

An important objective of the GOLF mission is to put some constraints on the amplitude of g-modes. If gravity modes have been already measured on white dwarfs, the detection is controversial in the solar case (van der Raay 1989). In this last case, the convective external layers largely limit their amplitudes, moreover, the superficial solar noise due to the supergranulation and the active regions is important in the range of frequencies where they are looked for (some $10^{-4} \mathrm{~Hz}$ ). Consequently, one needs a large integration time to disentangle the different processes. Moreover, the analysis of the solar noise would be facilitated by the determination of the shape and the deformations of the sodium line coupled with specific measurements on ground during the mission.

\subsection{Principle of the instrument}

The instrument is an optical resonance spectrometer which follows the variation of the Doppler velocity on the sodium absorption line. Such instrument has been using for a long time in Nice (Grec et al 1991). An entry filter selects a passing band of approximately $20 \AA$ bracketing the sodium $D_{1}$ and $\mathrm{D}_{2}$ lines located at 5896 and $5890 \AA$. Via an optical system, the entrance pupil is reimaged at the center of a gaseous sodium cell and the image of the Sun is rejecting at the infinity, which drastically limits the influence of the pointing. The photons absorbed by the sodium atoms are reemitted towards the two photomultipliers located at 90 degrees on both sides of the cell. The presence of a magnetic field around the cell will allow, via the Zeeman effect, to split the selected portion of the line $(25 \mathrm{~m} \AA)$. It is thus possible to choose, by modifying the circular polarization of the incident beam, a left or right portion of the line (fig 1a). The Doppler velocity will be deduced from the two dissymetric countings $\mathrm{N}_{1}$ and $\mathrm{N}_{2}$ as $\mathrm{V}=\mathrm{V}_{0}\left(\mathrm{~N}_{1}-\mathrm{N}_{2}\right) /\left(\mathrm{N}_{1}+\mathrm{N}_{2}\right)$ (fig.1b). By adding a modulation to the magnetic field, we investigate two 

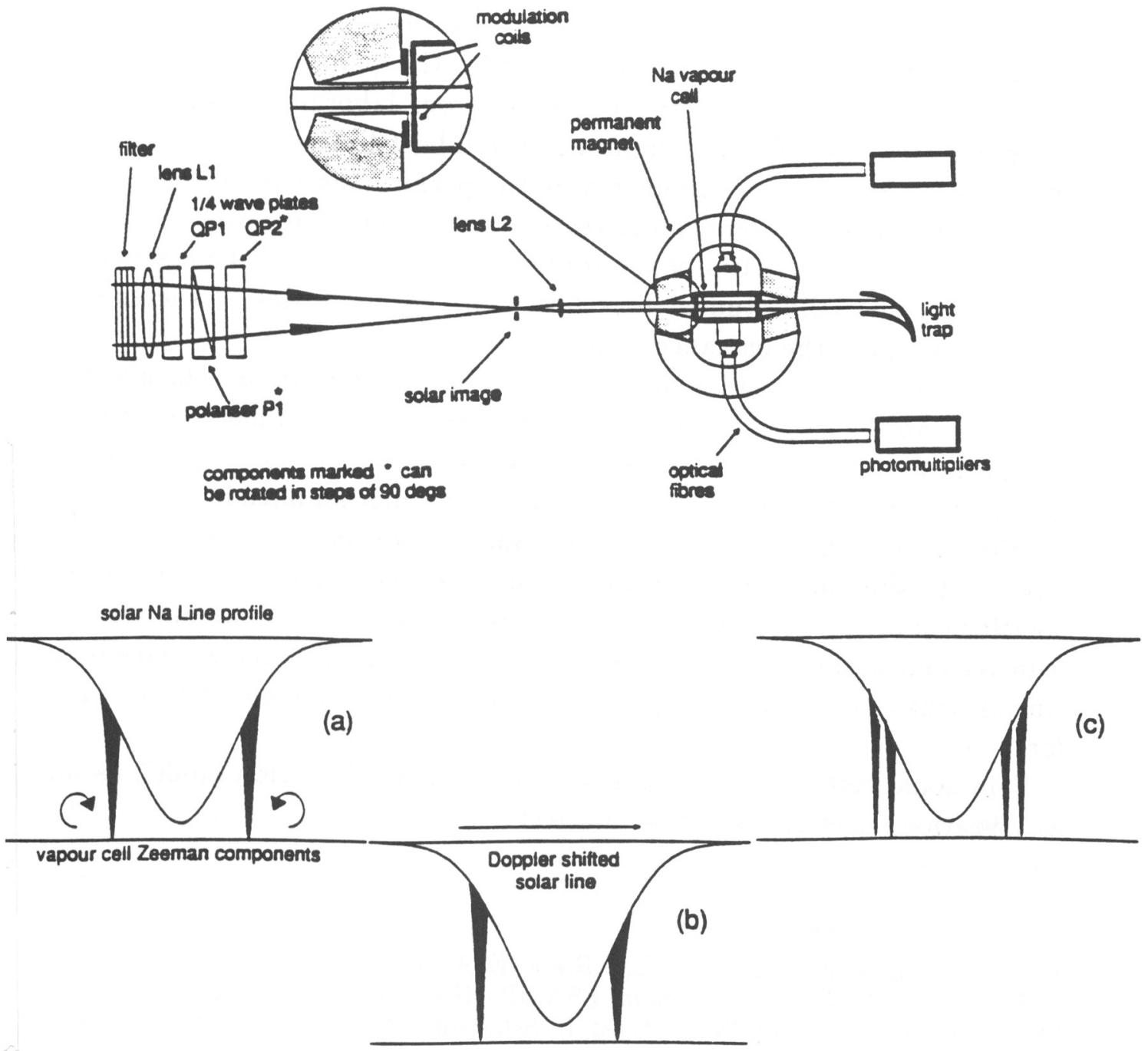

Fig. 26.1 Principle of the experiment and schematic design of the GOLF experiment

other bands, that makes possible to follow the shape and the distortions of the line and to determine $V_{0}$ (fig.1c).

\subsection{The present status}

The project is built under the responsability of IAS (Institut d'Astrophysique spatial) of Orsay (PI: A. Gabriel, PM: J. Charra), with the collaboration of IAC (Instituto de Astrofisica de Canarias) in Tenerife, the University of Bordeaux and Nice (PS: G. Grec), and the SAp (Service d'Astrophysique) in Saclay. Five models have been designed: the structural model, mechanically 
representative, the engineering model: electrically representative (these two models have been already delivered at ESA), the qualification model which is the first complete model and will stay in our laboratory and finally the flight and spare models. The flight model will be delivered at ESA at the end of the year 1993. The requirements on the performances of the experiment led to the realisation of original tests at the sub-system level. The thermal and optical properties of the sodium cell (the heart of the instrument) have been especially studied together with the properties of the spectral characteristics of the entrance sodium filter.

Concerning the detection chain, we have chosen phototubes which use the photon counting technique with a gain of $310^{6}$. The nominal counting rate would be $610^{6} / \mathrm{s}$ for each of the two photomultipliers in order to ensure the level of stability required to observe very small amplitude signal. This stability quality has been verified in laboratory during a three week acquisition with the nominal integration time of $4 \mathrm{~s}$. Using a filtered lamp in the wavelength of sodium, we have deduced from the counting rate an artificial velocity and performed its Fourier transform to garantee the absence of artificial peaks of the same amplitude than the signal we would like to look for.

Functional tests have been performed on the qualification model, as well as vibration, electrical and thermal tests.

\section{References}

Damé L. et al, ESA SP-286, ed. E.J. Rolfe (ESA Noordwijk 1988), p 367.

Gabriel A. et al., The Soho mission, ESA SP-1104, (1989), p. 13.

Turck-Chièze S., Däppen W., Provost J., Schatzman E. and Vignaud D., Phys. Rep 230, (1993) vol 2-4.

Turck-Chièze S. and Lopes I., ApJ., 408, (1993), 347.

Gough D. and Kosovishev, A.G., Mon. Not. R. Astron. Soc., 264 (1993), 522.

Turck-Chièze, H. Dzitko and I. Lopes, in Neutral Currents twenty years later,

Paris July 1993, to appear in World Scientific, ed. U. Nguyen-Khac

Grec G., Fossat E., Gelly B. and Smider F. X.,Solar Phys. 133, (1991), 13.

van der Raay H.B., in Progress of Seismology of the Sun and Stars, (1989),

Lecture Notes in Physics, 367, p 227. 